\title{
$\equiv Q$
}

\section{EUROPEAN RESPIRATORY journal}

FLAGSHIP SCIENTIFIC JOURNAL OF ERS

\section{Computerised respiratory sounds can differentiate smokers from non-smokers}

Ana Oliveira, Ipek Sen, Yasemin Kahya, Alda Marques

European Respiratory Journal 2016 48: PA4435; DOI: 10.1183/13993003.congress-2016.PA4435

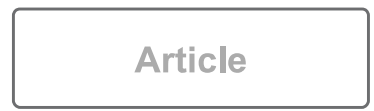

Figures \& Data

Info \& Metrics

\section{Abstract}

Cigarette smoking is associated with the development of respiratory diseases however, if diagnosed early, changes in the lung tissue may be reversible. Computerised respiratory sounds, namely crackles, are sensitive to detect changes within the lung tissue before other measures, however it is unknown if it is able to detect changes in the lungs of healthy smokers. This study investigated the differences in crackles between healthy smokers and non-smokers.

Healthy participants were recruited from a university campus. Respiratory sounds were recorded simultaneously at 4 chest locations (right and left anterior and posterior) using air-coupled electret microphones. Airflow (1.0-1.5 I/s) was recorded with a pneumotachograph. Breathing phases were detected using airflow signals and crackles (number and type-fine/coarse) with validated algorithms.

Thirty-two participants were enrolled: 19 non-smokers (25.1 $\pm 3.4 \mathrm{y} ; \mathrm{FEV}_{1} 101.7 \pm 11.6 \%$ predicted) and 13 smokers (24.1 $\pm 10 y ; F E V_{1} 102.5 \pm 8.2 \%$ predicted). Smokers presented significantly more inspiratory fine crackles $(p=0.010)$ at anterior regions and more expiratory fine crackles at posterior regions $(p=0.015)$ No other significant differences were found. 

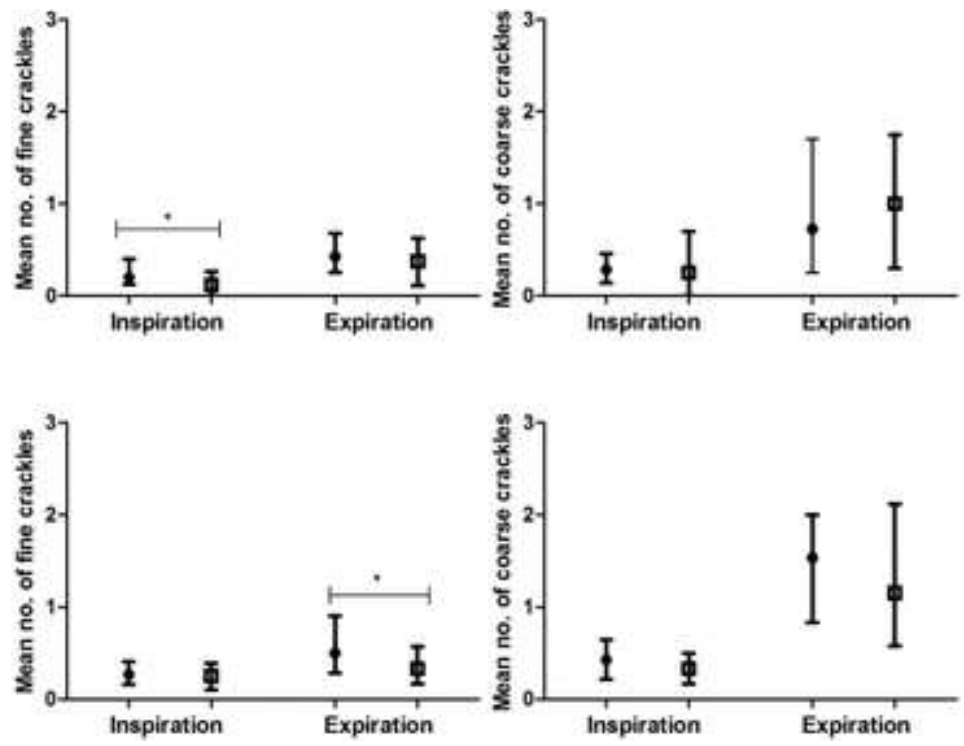

Download figure

Open in new tab

Download powerpoint

Smokers presented more fine crackles than non-smokers. Fine crackles are often the earliest sign of disease, often present before detection of changes in radiology. Thus, crackles might be a promising measure to early detect respiratory diseases in smokers.

Monitoring Spirometry Inflammation

\section{We recommend}

Comparison of adventitious respiratory sounds between stable COPD and AECOPD

Cristina Jácome et al., European Respiratory Journal

Crackles' sensitivity to pulmonary rehabilitation

Cristina Jácome et al., European Respiratory Journal

Computer aided lung sound analysis in smokers Mohammed Alzahrani et al., European Respiratory Journal

Lung function predicts lung cancer risk in smokers: a tool for targeting screening programmes.

E Calabrò et al., European Respiratory Journal
Majority of never-smokers with airflow limitation do not have asthma: the Copenhagen General Population Study

Yunus Çolak et al., Thorax

Wheezes, crackles and rhonchi: simplifying description of lung sounds increases the agreement on their classification: a study of 12 physicians' classification of lung sounds from video recordings Hasse Melbye et al., BMJ Open Resp Res

Sputum neutrophils are elevated in smelter workers, and systemic neutrophils are associated with rapid decline in FEV1

Johny Kongerud et al., Occup Environ Med 